\title{
Invariant subspaces of submarkovian semigroups
}

\author{
A. F. M. TER ELST ${ }^{1}$ AND DEREK W. ROBINSON ${ }^{2}$
}

Abstract. We characterize the invariance under a submarkovian semigroup of a measurable subset by capacity conditions on its boundary.

\section{Introduction}

Let $S$ be a submarkovian semigroup acting on $L_{2}(X ; \mu)$ where $X$ is a locally compact $\sigma$-compact metric space and $\mu$ a Radon measure with supp $\mu=X$ (see [7] and [3]). If $\Omega$ is a measurable subset of $X$ we give characterizations of the $S$-invariance of $L_{2}(\Omega)$, i.e. the property $S_{t} L_{2}(\Omega) \subseteq L_{2}(\Omega)$ for all $t>0$. This corresponds to $S$-invariance of the set $\Omega$ in the terminology of [7], Section 1.6.

If one interprets $S$ as describing a dissipative evolution then the invariance of $\Omega$ corresponds to impenetrability of the boundary $\partial \Omega$. Therefore one would expect the invariance to be characterized by properties of the boundary. Our main result establishes that this is indeed the case; invariance of $\Omega$ is equivalent to a capacity condition on the boundary $\partial \Omega$. In this respect it differs from the standard characterizations of invariance. These are either in terms of local properties of the generator of $S$ (see, for example, [7], Section 1.6 and [10], Section 2.1) or, in the case of irreducibility of the semigroup, in terms of spectral properties or algebraic properties (see [11], Section XIII.12, and [12], Proposition 2.1).

In order to formulate our main result we must first introduce some basic definitions and notation.

Let $H$ denote the positive self-adjoint generator of $S$ and $h$ the corresponding Dirichlet form (see [7] and [3]). We assume that $h$ is regular in the sense of [7], i.e. $D(h) \cap C_{c}(X)$ is dense in $D(h)$ with respect to the graph norm $\varphi \mapsto\|\varphi\|_{D(h)}=\left(h(\varphi)+\|\varphi\|_{2}^{2}\right)^{1 / 2}$ and also dense in $C_{0}(X)$ with respect to the uniform norm. Moreover, we define $h$ to be local if $h(\varphi, \psi)=0$ for all $\varphi, \psi \in D(h)$ with $\varphi \psi=0$. This notion appears slightly stronger than locality as defined in [7] but it is in fact equivalent by a result of Schmuland [13]. Nevertheless, it is weaker than the form of locality introduced in [3]. Next if $\Omega$ is a subset of $X$ and $A \subseteq \bar{\Omega}$ then we define $\operatorname{cap}_{\Omega}(A) \in[0, \infty]$ by

Mathematics Subject Classifications (2000): 28A12, 31C15, 47A35.

Key words: invariance, capacity, Dirichlet form. 


$$
\begin{array}{r}
\operatorname{cap}_{\Omega}(A)=\inf \left\{\|\varphi\|_{D(h)}^{2}: \varphi \in D(h) \text { and there exists an open } V \subset X\right. \\
\text { such that } A \subset V \text { and } \varphi \geq 1 \text { a.e. on } V \cap \Omega\} .
\end{array}
$$

If there is any possible ambiguity then we use the notation $\operatorname{cap}_{\Omega, h}(A)$ instead of $\operatorname{cap}_{\Omega}(A)$. Note that $\Omega \mapsto \operatorname{cap}_{\Omega}(A)$ is a monotonically increasing function for each fixed $A$. Moreover, $\operatorname{cap}_{X}(A)=\operatorname{cap}(A)$ where $\operatorname{cap}(A)$ is the usual capacity of $A$ with respect to $h$ as defined in [7], Section 2.1, or [3], Section 1.8. A similar definition of relative capacity is introduced in [1] [2] and in special cases the two definitions coincide, e.g. if $H$ is the Laplacian on $\mathbf{R}^{d}$ and $\Omega$ is a bounded set with Lipschitz boundary. We do not know, however, whether the definitions coincide in general or whether our results are valid when reformulated in terms of this alternative definition.

The main result of this note is the following statement.

THEOREM 1.1. Let $\Omega$ be a measurable subset of $X$ and suppose that the regular Dirichlet form $h$ is local. The following conditions are equivalent.

I. $S_{t} L_{2}(\Omega) \subseteq L_{2}(\Omega)$ for all $t>0$.

II. There exist $A_{1}, A_{2} \subseteq \partial \Omega$ such that $\partial \Omega=A_{1} \cup A_{2}$ and $\operatorname{cap}_{\Omega}\left(A_{1}\right)=0=\operatorname{cap}_{\Omega^{\mathrm{c}}}\left(A_{2}\right)$.

The proof of the implication $\mathrm{I} \Rightarrow \mathrm{II}$ in Theorem 1.1 does not require locality of $h$ but this property is used in proving the converse. In the course of the proof we also derive several alternative characterizations of the $S$-invariance of $L_{2}(\Omega)$. Another characterization of $S$-invariance for general measurable subsets $\Omega$ is given in [5], Theorem 1.3.

Theorem 1.1 extends to local regular Dirichlet forms a result of [12] for forms defined by degenerate elliptic operators on $\mathbf{R}^{d}$ and open subsets $\Omega$. Theorem 1.1 in [12] states that if the coefficients of the degenerate operator are Lipschitz continuous and $\Omega$ is a Lipschitz domain then $S$-invariance of $L_{2}(\Omega)$ is equivalent to cap $(\partial \Omega)=0$. Nevertheless it is straightforward to construct examples of $S$-invariant subspaces $L_{2}(\Omega)$ with $|\partial \Omega|>0$. But then $\operatorname{cap}(\partial \Omega) \geq|\partial \Omega|>0$. So the condition $\operatorname{cap}(\partial \Omega)=0$ does not give a characterization of $S$-invariance in general. The current results show that $S$-invariance of the subspace $L_{2}(\Omega)$ can indeed be characterized by conditions of small capacity of sets close to the boundary of $\Omega$ without any detailed assumptions of regularity.

In the last section we illustrate our results with various examples of degenerate elliptic operators. Note that for degenerate operators a general sufficient criterion for the existence of invariant subspaces was given in [6], Lemma 6.4 and Proposition 6.10.

\section{Invariance criteria}

We begin with a useful corollary of a theorem of Ouhabaz valid for 'local' accretive closed sesquilinear forms. 
PROPOSITION 2.1. Let $\mathfrak{a}$ be an accretive closed sesquilinear form in a Hilbert space $L_{2}(Y)$, where $Y$ is a measure space. Suppose that $\mathfrak{a}(\varphi, \psi)=0$ for all $\varphi, \psi \in D(\mathfrak{a})$ with $\varphi \psi=0$. Let $T$ be the semigroup associated to $\mathfrak{a}$ and let $\Omega$ be a measurable subset of $Y$. Then the following are equivalent.

I. $T_{t} L_{2}(\Omega) \subseteq L_{2}(\Omega)$ for all $t>0$.

II. $\mathbb{1}_{\Omega} \varphi \in D(\mathfrak{a})$ for all $\varphi \in D(\mathfrak{a})$.

III. There exists a core $D$ for $\mathfrak{a}$ such that $\mathbb{1}_{\Omega} \varphi \in D(\mathfrak{a})$ for all $\varphi \in D$.

Proof. Let $P$ be the orthogonal projection of $L_{2}(Y)$ onto $L_{2}(\Omega)$. By [10], Theorem 2.2, the invariance of $L_{2}(\Omega)$ under $T$ is equivalent to the statement(s) that there exists a core $D$ for $\mathfrak{a}$ (or for every core) such that $P(D) \subseteq D(\mathfrak{a})$ and $\operatorname{Re} \mathfrak{a}(P \varphi, \varphi-P \varphi) \geq 0$ for all $\varphi \in D$. This implies that $\mathrm{I} \Rightarrow \mathrm{II} \Rightarrow \mathrm{III}$. But $P \varphi=\mathbb{1}_{\Omega} \varphi$ and $\varphi-P \varphi=\mathbb{1}_{\Omega^{\mathrm{c}}} \varphi$. So if $P(D) \subseteq D(\mathfrak{a})$ then by 'locality' $\mathfrak{a}(P \varphi, \varphi-P \varphi)=0$ for all $\varphi \in D$. This proves the proposition.

As a preliminary for the proof of Theorem 1.1 we derive some criteria for the $S$ invariance property. The first result is an implication of invariance which does not require locality of the form $h$.

PROPOSITION 2.2. Let $\Omega$ be a measurable subset of $X$. If $S_{t} L_{2}(\Omega) \subseteq L_{2}(\Omega)$ for all $t>0$ then for all $\varepsilon>0$ there exist open sets $U, V_{1}, V_{2} \subseteq X$ such that $\operatorname{cap}(U)<\varepsilon$, $\partial \Omega \subseteq V_{1} \cup V_{2},\left|V_{1} \cap \Omega \cap U^{\mathrm{c}}\right|=0$ and $\left|V_{2} \cap \Omega^{\mathrm{c}} \cap U^{\mathrm{c}}\right|=0$.

Proof. Let $W$ be a relatively compact open subset of $X$. We first show that for all $\varepsilon>0$ there exists an open set $U \subseteq X$ such that $\operatorname{cap}(U)<\varepsilon$ and for all $x \in W \cap \partial \Omega$ there exists a $\delta>0$ such that $\left|B(x ; \delta) \cap \Omega \cap U^{\mathrm{c}}\right|=0$ or $\left|B(x ; \delta) \cap \Omega^{\mathrm{c}} \cap U^{\mathrm{c}}\right|=0$.

Since $h$ is regular there exists a $\varphi \in D(h) \cap C_{c}(X)$ with $0 \leq \varphi \leq 1$ and $\left.\varphi\right|_{\bar{W}}=1$. Set $\psi=\varphi \mathbb{1}_{\Omega}$. Then since $L_{2}(\Omega)$ is $S$-invariant it follows, by [7], Theorem 1.6.1, that $\psi \in D(h)$. Let $\widetilde{\psi}$ be a quasi-continuous representative of $\psi$. Then there exists an open set $U \subseteq X$ such that $\operatorname{cap}(U)<\varepsilon$ and $\left.\widetilde{\psi}\right|_{U^{\text {c }}}$ is continuous. Then

$$
\left.\widetilde{\psi}\right|_{W \cap \Omega \cap U^{c}}=\left.\psi\right|_{W \cap \Omega \cap U^{c}}=1 \text { a.e. }
$$

and

$$
\left.\widetilde{\psi}\right|_{W \cap \Omega^{\mathrm{c}} \cap U^{\mathrm{c}}}=\left.\psi\right|_{W \cap \Omega^{\mathrm{c}} \cap U^{\mathrm{c}}}=0 \text { a.e. }
$$

by construction of $\varphi$ and $\psi$.

Let $x \in W \cap \partial \Omega$. If $x \in U$ then clearly there exists a $\delta>0$ such that $B(x ; \delta) \subseteq U$. Hence $\left|B(x ; \delta) \cap \Omega \cap U^{\mathrm{c}}\right|=0$. So suppose that $x \in U^{\mathrm{c}}$. Since $\left.\widetilde{\psi}\right|_{U^{\mathrm{c}}}$ is continuous at $x$ there exists a $\delta>0$ such that

$$
|\widetilde{\psi}(y)-\widetilde{\psi}(x)|<\frac{1}{2}
$$


for all $y \in B(x ; \delta) \cap U^{\mathrm{c}}$. We may assume that $B(x ; \delta) \subseteq W$. Since $B(x ; \delta) \cap \Omega \cap U^{\mathrm{c}} \subseteq$ $W \cap \Omega \cap U^{\mathrm{c}}$ it follows that $\widetilde{\psi}(y)=1$ for a.e. $y \in B(x ; \delta) \cap \Omega \cap U^{\mathrm{c}}$. Similarly $\widetilde{\psi}(y)=0$ for a.e. $y \in B(x ; \delta) \cap \Omega^{\mathrm{c}} \cap U^{\mathrm{c}}$. Hence if $\left|B(x ; \delta) \cap \Omega \cap U^{\mathrm{c}}\right|>0$ then it follows from (2.1) that $\left|B(x ; \delta) \cap \Omega^{\mathrm{c}} \cap U^{\mathrm{c}}\right|=0$.

Since $X$ is $\sigma$-compact, for all $\varepsilon>0$ there exists an open set $U \subseteq X$ such that $\operatorname{cap}(U)<\varepsilon$ and for all $x \in \partial \Omega$ there exists $\delta_{x}>0$ with $\left|B\left(x ; \delta_{x}\right) \cap \Omega \cap U^{\mathrm{c}}\right|=0$ or $\left|B\left(x ; \delta_{x}\right) \cap \Omega^{\mathrm{c}} \cap U^{\mathrm{c}}\right|=$ 0. Define

$$
A_{1}=\left\{x \in \partial \Omega:\left|B\left(x ; \delta_{x}\right) \cap \Omega \cap U^{\mathrm{c}}\right|=0\right\}
$$

and

$$
A_{2}=\left\{x \in \partial \Omega:\left|B\left(x ; \delta_{x}\right) \cap \Omega^{\mathrm{c}} \cap U^{\mathrm{c}}\right|=0\right\} .
$$

By the basic covering theorem, [8] Theorem 1.2, there exists a subset $A_{1}^{\prime} \subseteq A_{1}$ such that $\bigcup_{x \in A_{1}} B\left(x ; 5^{-1} \delta_{x}\right) \subseteq \bigcup_{x \in A_{1}^{\prime}} B\left(x ; \delta_{x}\right)$ and the sets $B\left(x ; 5^{-1} \delta_{x}\right)$ with $x \in A_{1}^{\prime}$ are pairwise disjoint. Since $X$ is $\sigma$-compact, it is separable. Therefore $A_{1}^{\prime}$ is countable. Set $V_{1}=\bigcup_{x \in A_{1}^{\prime}} B\left(x ; \delta_{x}\right)$. Then $V_{1}$ is open in $X$ and $A_{1} \subseteq \bigcup_{x \in A_{1}} B\left(x ; 5^{-1} \delta_{x}\right) \subseteq V_{1}$. Moreover,

$$
\left|V_{1} \cap \Omega \cap U^{\mathrm{c}}\right| \leq \sum_{x \in A_{1}^{\prime}}\left|B\left(x ; \delta_{x}\right) \cap \Omega \cap U^{\mathrm{c}}\right|=0 .
$$

Similarly there exists an open $V_{2} \subseteq X$ such that $A_{2} \subseteq V_{2}$ and $\left|V_{2} \cap \Omega^{\mathrm{c}} \cap U^{\mathrm{c}}\right|=0$. Finally, $\partial \Omega=A_{1} \cup A_{2} \subseteq V_{1} \cup V_{2}$.

The implication $\mathrm{I} \Rightarrow \mathrm{II}$ in Theorem 1.1 follows from Proposition 2.2 and the next proposition. We emphasize that the following proposition does not require locality of the form $h$.

PROPOSITION 2.3. Let $\Omega$ be a measurable subset of $X$. If for all $\varepsilon>0$ there exist open sets $U, V_{1}, V_{2} \subseteq X$ such that $\operatorname{cap}(U)<\varepsilon, \partial \Omega \subseteq V_{1} \cup V_{2},\left|V_{1} \cap \Omega \cap U^{\mathrm{c}}\right|=0$ and $\left|V_{2} \cap \Omega^{\mathrm{c}} \cap U^{\mathrm{c}}\right|=0$, then there exist $A_{1}, A_{2} \subseteq \partial \Omega$ such that $\partial \Omega=A_{1} \cup A_{2}$ and $\operatorname{cap}_{\Omega}\left(A_{1}\right)=0=\operatorname{cap}_{\Omega^{\mathrm{c}}}\left(A_{2}\right)$.

Proof. By assumption for all $n \in \mathbf{N}$ there exist open sets $U_{n}, V_{1 n}, V_{2 n} \subseteq X$ such that $\operatorname{cap}\left(U_{n}\right)<2^{-n}, \partial \Omega \subseteq V_{1 n} \cup V_{2 n},\left|V_{1 n} \cap \Omega \cap U_{n}^{\mathrm{c}}\right|=0$ and $\left|V_{2 n} \cap \Omega^{\mathrm{c}} \cap U_{n}^{\mathrm{c}}\right|=0$. Then $\operatorname{cap}\left(\bigcup_{k=n}^{\infty} U_{k}\right)<2^{-n+1}$,

$$
\left|\left(\bigcup_{k=n}^{\infty} V_{1 k}\right) \cap \Omega \cap\left(\bigcup_{k=n}^{\infty} U_{k}\right)^{\mathrm{c}}\right| \leq \sum_{k=n}^{\infty}\left|V_{1 k} \cap \Omega \cap U_{k}^{\mathrm{c}}\right|=0
$$

and a similar expression involving the $V_{2 k}$, for all $n \in \mathbf{N}$. So without loss of generality we may assume that $U_{1} \supseteq U_{2} \supseteq \ldots, V_{11} \supseteq V_{12} \supseteq \ldots$ and $V_{21} \supseteq V_{22} \supseteq \ldots$ Define

$$
A_{1}=\bigcap_{n=1}^{\infty} V_{1 n} \cap \partial \Omega \text { and } A_{2}=\bigcap_{n=1}^{\infty} V_{2 n} \cap \partial \Omega \text {. }
$$


Let $x \in \partial \Omega$ and suppose that $x \notin A_{1}$. Then there exists an $n \in \mathbf{N}$ such that $x \notin V_{1 n}$. Therefore $x \notin V_{1 k}$ for all $k \geq n$. Since $\partial \Omega \subseteq V_{1 k} \cup V_{2 k}$ for all $k \in \mathbf{N}$ it follows that $x \in V_{2 k}$ for all $k \geq n$ and $x \in A_{2}$. Therefore $\partial \Omega=A_{1} \cup A_{2}$.

Finally, let $n \in \mathbf{N}$. Since $\operatorname{cap}\left(U_{n}\right)<2^{-n}$ there exists a $\varphi \in D(h)$ such that $\varphi \geq 1$ a.e. on $U_{n}$ and $\|\varphi\|_{D(h)}^{2}<2^{-n}$. Because $\left|V_{1 n} \cap \Omega \cap U_{n}^{\mathrm{c}}\right|=0$ it follows that $\varphi \geq 1$ a.e. on $V_{1 n} \cap \Omega$. So $\operatorname{cap}_{\Omega}\left(A_{1}\right)=0$. Similarly, $\operatorname{cap}_{\Omega^{\mathrm{c}}}\left(A_{2}\right)=0$.

If the form $h$ is local then there is also a converse of Propositions 2.2 and 2.3. In fact there is even a seemingly weaker version. The next theorem amplifies the characterizations of Theorem 1.1.

THEOREM 2.4. Let $\Omega$ be a measurable subset of $X$ and suppose that the regular Dirichlet form $h$ is local. Then the following conditions are equivalent.

I. $S_{t} L_{2}(\Omega) \subseteq L_{2}(\Omega)$ for all $t>0$.

II. For all $\varepsilon>0$ there exist open sets $U, V_{1}, V_{2} \subseteq X$ such that $\operatorname{cap}(U)<\varepsilon, \partial \Omega \subseteq$ $V_{1} \cup V_{2},\left|V_{1} \cap \Omega \cap U^{\mathrm{c}}\right|=0$ and $\left|V_{2} \cap \Omega^{\mathrm{c}} \cap U^{\mathrm{c}}\right|=0$.

III. There exist $A_{1}, A_{2} \subseteq \partial \Omega$ such that $\partial \Omega=A_{1} \cup A_{2}$ and $\operatorname{cap}_{\Omega}\left(A_{1}\right)=0=\operatorname{cap}_{\Omega^{c}}\left(A_{2}\right)$.

IV. There exists an $M>0$ such that for all $\varepsilon>0$ there exist open sets $V_{1}, V_{2} \subseteq X$ and a function $\psi \in D(h)$ such that $\partial \Omega \subseteq V_{1} \cup V_{2}, h(\psi) \leq M,\|\psi\|_{2}<\varepsilon$ and $\psi=1$ a.e. on $\left(V_{1} \cap \Omega\right) \cup\left(V_{2} \cap \Omega^{\mathrm{c}}\right)$.

Proof. The implication $\mathrm{I} \Rightarrow \mathrm{II}$ follows from Proposition 2.2, the implication $\mathrm{II} \Rightarrow \mathrm{III}$ by Proposition 2.3 and the implication III $\Rightarrow$ IV from the estimate $\|\varphi \vee \psi\|_{D(h)}^{2} \leq\|\varphi\|_{D(h)}^{2}+$ $\|\psi\|_{D(h)}^{2}$ for all $\varphi, \psi \in D(h)$ (see [3], Lemma 8.1.2.1).

'IV $\Rightarrow \mathrm{I}$ '. Let $\varphi \in D(h) \cap C_{c}(X)$ and let $M>0$ be as in Condition 2.4. Let $n \in \mathbf{N}$. By assumption there exist open sets $V_{1 n}, V_{2 n} \subseteq X$ and a function $\psi_{n} \in D(h)$ such that $\partial \Omega \subseteq V_{1 n} \cup V_{2 n}, h\left(\psi_{n}\right) \leq M,\left\|\psi_{n}\right\|_{2} \leq n^{-1}$ and $\psi_{n}=1$ a.e. on $\left(V_{1 n} \cap \Omega\right) \cup\left(V_{2 n} \cap \Omega^{\mathrm{c}}\right)$. We may assume that $0 \leq \psi_{n} \leq 1$. Set

$$
\varphi_{n}=\left(\varphi-\varphi \psi_{n}\right) \mathbb{1}_{\Omega} .
$$

We shall prove that $\varphi_{n} \in D(h)$.

Set $K=\operatorname{supp} \varphi$. Define

$$
K_{10}=K \cap \Omega \cap V_{1 n}^{\mathrm{c}}, K_{20}=K \cap \Omega^{\mathrm{c}} \cap V_{2 n}^{\mathrm{c}},
$$

$K_{1}=\overline{K_{10}}$ and $K_{2}=\overline{K_{20}}$. Then $K_{1}$ and $K_{2}$ are compact. We next show that $K_{1}$ and $K_{2}$ are disjoint. Let $x \in K_{1} \cap K_{2}$. Then $x \in \bar{\Omega} \cap \overline{\Omega^{\mathrm{c}}}=\partial \Omega \subseteq V_{1 n} \cup V_{2 n}$. Suppose $x \in V_{1 n}$. Now $x \in K_{1}=\overline{K_{10}}$, so $V_{1 n} \cap K_{10} \neq \emptyset$. This is a contradiction. Similarly $x \in V_{2 n}$ gives a contradiction. Hence $K_{1}$ and $K_{2}$ are disjoint. Since $h$ is regular there exists 
a $\chi_{n} \in D(h) \cap C_{c}(X)$ such that $\left.\chi_{n}\right|_{K_{1}}=1$ and $\left.\chi_{n}\right|_{K_{2}}=0$. In particular $\chi_{n}(x)=1$ for all $x \in K_{10}$ and $\chi_{n}(x)=0$ for all $x \in K_{20}$. Note that

$$
K \cap\left(\left(V_{1 n} \cap \Omega\right) \cup\left(V_{2 n} \cap \Omega^{\mathrm{c}}\right)\right)^{\mathrm{c}} \cap \Omega=K_{10}
$$

and

$$
K \cap\left(\left(V_{1 n} \cap \Omega\right) \cup\left(V_{2 n} \cap \Omega^{\mathrm{c}}\right)\right)^{\mathrm{c}} \cap \Omega^{\mathrm{c}}=K_{20} .
$$

Hence it follows that $\varphi_{n}=\left(\varphi-\varphi \psi_{n}\right) \chi_{n}$ a.e. So $\varphi_{n} \in D(h)$.

Since $\varphi-\varphi \psi_{n} \in D(h)$ and $\varphi_{n} \in D(h)$ it follows that $\left(\varphi-\varphi \psi_{n}\right) \mathbb{1}_{\Omega^{c}} \in D(h)$. Then, by locality of $h$,

$$
h\left(\varphi-\varphi \psi_{n}\right)=h\left(\left(\varphi-\varphi \psi_{n}\right) \mathbb{1}_{\Omega}\right)+h\left(\left(\varphi-\varphi \psi_{n}\right) \mathbb{1}_{\Omega^{\mathrm{c}}}\right) \geq h\left(\varphi_{n}\right)
$$

Moreover,

$$
h\left(\varphi \psi_{n}\right)^{1 / 2} \leq h(\varphi)^{1 / 2}\left\|\psi_{n}\right\|_{\infty}+\|\varphi\|_{\infty} h\left(\psi_{n}\right)^{1 / 2} \leq h(\varphi)^{1 / 2}+M^{1 / 2}\|\varphi\|_{\infty}
$$

for all $n \in \mathbf{N}$. So the sequence $\varphi_{1}, \varphi_{2}, \ldots$ is bounded in $D(h)$. Therefore this sequence has a weakly convergent subsequence. Passing to a subsequence, if necessary, there exists a $\hat{\varphi} \in D(h)$ such that $\lim _{n \rightarrow \infty} \varphi_{n}=\hat{\varphi}$ weakly in $D(h)$. Then $\lim _{n \rightarrow \infty} \varphi_{n}=\hat{\varphi}$ weakly in $L_{2}(X)$. But $\lim _{n \rightarrow \infty} \varphi \psi_{n}=0$ in $L_{2}(X)$. So

$$
\lim _{n \rightarrow \infty} \varphi_{n}=\lim _{n \rightarrow \infty}\left(\varphi-\varphi \psi_{n}\right) \mathbb{1}_{\Omega}=\varphi \mathbb{1}_{\Omega}
$$

in $L_{2}(X)$. Therefore $\varphi \mathbb{1}_{\Omega}=\hat{\varphi} \in D(h)$. Now the Statement I follows from Propostion 2.1 since $D(h) \cap C_{c}(X)$ is a core for $h$.

A combination of Theorem 1.1 and Proposition 2.1 gives the following corollary.

COROLLARY 2.5. Let $\Omega$ be a measurable subset of $X$ and suppose that the regular Dirichlet form $h$ is local. The following conditions are equivalent.

I. $\mathbb{1}_{\Omega} \varphi \in D(h)$ for all $\varphi \in D(h)$.

II. There exist $A_{1}, A_{2} \subseteq \partial \Omega$ such that $\partial \Omega=A_{1} \cup A_{2}$ and $\operatorname{cap}_{\Omega}\left(A_{1}\right)=0=\operatorname{cap}_{\Omega^{\mathrm{c}}}\left(A_{2}\right)$.

We end this section with some remarks concerning comparison with the ordinary capacity.

COROLLARY 2.6. Suppose the regular Dirichlet form $h$ is local. If $\operatorname{cap}(\partial \Omega)=0$ then $L_{2}(\Omega)$ is $S$-invariant.

Proof. The condition $\operatorname{cap}(\partial \Omega)=0$ clearly implies Condition II of Theorem 1.1. 
This corollary extends to regular local Dirichlet forms an earlier result in [12] valid for forms defined by degenerate elliptic operators on $\mathbf{R}^{d}$. In fact Theorem 2.4 gives the following improvement.

COROLLARY 2.7. Let $\Omega$ be a measurable subset of $X$ and suppose that the regular Dirichlet form $h$ is local. Let $M \in \mathbf{R}$. Suppose for all $\varepsilon>0$ there exists a $\psi \in D(h)$ such that $h(\psi) \leq M,\|\psi\|_{2}<\varepsilon$ and $\psi \geq 1$ on a neighbourhood of $\partial \Omega$. Then $S_{t} L_{2}(\Omega) \subseteq L_{2}(\Omega)$ for all $t>0$.

The space $L_{2}(\Omega)$ is clearly unchanged if $\Omega$ is modified by a set of measure zero. Therefore the $S$-invariance, Condition I of Theorem 1.1, is not sensitive to modifications of this type. Alternatively, if $\Omega_{1}$ and $\Omega_{2}$ are two measurable subsets of $X$ with $\overline{\Omega_{1}}=\overline{\Omega_{2}}$ and $\left|\Omega_{1} \Delta \Omega_{2}\right|=0$ then $\operatorname{cap}_{\Omega_{1}}(A)=\operatorname{cap}_{\Omega_{2}}(A)$ for all $A \subseteq \overline{\Omega_{1}}$. This follows immediately from the definition. On the other hand, the boundaries of $\Omega_{1}$ and $\Omega_{2}$ may be different. The capacity assumptions of Condition II of the theorem are therefore more sensitive to variations of $\Omega$ since there are sets of measure zero which have strictly positive capacity. Nevertheless these assumptions do not depend necessarily on the entire boundary of $\Omega$.

An open set $\Omega$ is called topologically regular if $\Omega$ is equal to the interior of its closure, i.e. if $\Omega=\stackrel{\circ}{\Omega}$ or, equivalently, if $\partial \Omega=\partial\left(\bar{\Omega}^{\mathrm{c}}\right)$. For any set $\Omega$ the set $\stackrel{\circ}{\Omega}$ is topologically regular. If $\Omega$ is a measurable subset of $X$ with $|\partial \Omega|=0$ then $\partial \stackrel{\circ}{\Omega} \subseteq \partial \bar{\Omega} \subseteq \partial \Omega$, so $|\partial \stackrel{\circ}{\bar{\Omega}}|=|\partial \bar{\Omega}|=0$. Hence $L_{2}(\Omega)=L_{2}(\bar{\Omega})=L_{2}(\stackrel{\circ}{\Omega})$. Therefore $L_{2}(\Omega)$ is $S$-invariant if and only if $L_{2}(\stackrel{\circ}{\Omega})$ is also $S$-invariant. Next, note that if in addition $\Omega$ is open then $\Omega \subseteq \stackrel{\circ}{\Omega}$ and

$$
\partial \Omega=\partial \stackrel{\circ}{\Omega} \cup(\stackrel{\circ}{\Omega} \backslash \Omega) .
$$

Moreover, by definition of $\operatorname{cap}_{\Omega^{\mathrm{c}}}(A)$ it follows (with $V=\stackrel{\circ}{\bar{\Omega}}$ and $\varphi=0$ ) that $\left.\operatorname{cap}_{\Omega^{\mathrm{c}}} \stackrel{\circ}{(\Omega} \backslash \Omega\right)=$ 0 , although in general $\operatorname{cap}(\stackrel{\circ}{\Omega} \backslash \Omega) \neq 0$. (See Example 3.2) Consequently one has the following extension of Theorem 1.1 for open sets $\Omega$.

COROLLARY 2.8. Let $\Omega$ be an open subset of $X$ with $|\partial \Omega|=0$ and suppose that the regular Dirichlet form $h$ is local. The following conditions are equivalent.

I. $S_{t} L_{2}(\Omega) \subseteq L_{2}(\Omega)$ for all $t>0$.

II. There exist $A_{1}, A_{2} \subseteq \partial \Omega$ such that $A_{1} \cup A_{2}=\partial \stackrel{\circ}{\bar{\Omega}}$ and $\operatorname{cap}_{\Omega}\left(A_{1}\right)=0=\operatorname{cap}_{\bar{\Omega}^{c}}\left(A_{2}\right)$.

Note that in Condition II the open set $\bar{\Omega}^{\mathrm{c}}$ is used instead of $\Omega^{\mathrm{c}}$.

The sets $A_{1}$ and $A_{2}$ in Theorem 1.1 might have a non-empty intersection and we next prove that the capacity of $A_{1} \cap A_{2}$ vanishes. This is a consequence of a more general statement which again does not require locality of the Dirichlet form $h$. Note that a condition on $\left|A \backslash\left(\Omega_{1} \cup \Omega_{2}\right)\right|$ is necessary in the next lemma since cap $(B) \geq|B|$ for any measurable set. 
LEMMA 2.9. Let $\Omega_{1}, \Omega_{2}$ be two subsets of $X$ and let $A \subseteq \overline{\Omega_{1}} \cap \overline{\Omega_{2}}$. Suppose that $\operatorname{cap}_{\Omega_{1}}(A)=\operatorname{cap}_{\Omega_{2}}(A)=0$. Moreover, suppose that there exists an open set $U$ in $X$ such that $A \subseteq U$ and $\left|U \backslash\left(\Omega_{1} \cup \Omega_{2}\right)\right|=0$. Then $\operatorname{cap}(A)=0$.

Proof. Let $\varepsilon>0$. There exist open $V_{1}, V_{2}$ in $X$ and $\varphi_{1}, \varphi_{2} \in D(h)$ such that $\left\|\varphi_{1}\right\|_{D(h)}^{2}<\varepsilon$, $\left\|\varphi_{2}\right\|_{D(h)}^{2}<\varepsilon, A \subseteq V_{1}, A \subseteq V_{2}, \varphi_{1} \geq 1$ a.e. on $V_{1} \cap \Omega_{1}$ and $\varphi_{2} \geq 1$ a.e. on $V_{2} \cap \Omega_{2}$. Then $\left\|\varphi_{1} \vee \varphi_{2}\right\|_{D(h)}^{2}<2 \varepsilon, V_{1} \cap V_{2} \cap U$ is open, $A \subseteq V_{1} \cap V_{2} \cap U$ and $\varphi_{1} \vee \varphi_{2} \geq 1$ a.e. on $\left(V_{1} \cap \Omega_{1}\right) \cup\left(V_{2} \cap \Omega_{2}\right)$. Clearly $\varphi_{1} \vee \varphi_{2} \geq 1$ a.e. on the null set $U \backslash\left(\Omega_{1} \cup \Omega_{2}\right)$. But

$$
V_{1} \cap V_{2} \cap U \subseteq\left(V_{1} \cap \Omega_{1}\right) \cup\left(V_{2} \cap \Omega_{2}\right) \cup\left(U \backslash\left(\Omega_{1} \cup \Omega_{2}\right)\right)
$$

Therefore $\varphi_{1} \vee \varphi_{2} \geq 1$ a.e. on $V_{1} \cap V_{2} \cap U$. Consequently cap $\left(V_{1} \cap V_{2} \cap U\right)<2 \varepsilon$. Hence $\operatorname{cap}(A)=0$.

COROLLARY 2.10. Let $\Omega$ be a subset of $X$ and let $A \subseteq \partial \Omega$ be such that $\operatorname{cap}_{\Omega}(A)=$ $0=\operatorname{cap}_{\Omega^{\mathrm{c}}}(A)$. Then $\operatorname{cap}(A)=0$.

Finally we establish a type of domination for invariant subspaces.

COROLLARY 2.11. Let $h, k$ be two regular Dirichlet forms on $X$ with $k \leq h$ and $k$ local. Further let $S, T$ be the associated semigroups on $L_{2}(X)$ and $\Omega$ a measurable subset of $X$. If $L_{2}(\Omega)$ is $S$-invariant then $L_{2}(\Omega)$ is $T$-invariant.

Proof. It follows by definition of the order relation for quadratic forms that $D(h) \subseteq D(k)$ and $k(\varphi) \leq h(\varphi)$ for all $\varphi \in D(h)$. Therefore if $W$ is a subset of $X$ and $A \subseteq \bar{W}$ then $\operatorname{cap}_{W, k}(A) \leq \operatorname{cap}_{W, h}(A)$.

It follows from Propositions 2.2 and 2.3 that there exist $A_{1}, A_{2} \subseteq \partial \Omega$ such that $\partial \Omega=$ $A_{1} \cup A_{2}$ and $\operatorname{cap}_{\Omega, h}\left(A_{1}\right)=0=\operatorname{cap}_{\Omega^{\mathrm{c}}, h}\left(A_{2}\right)$. Therefore $\operatorname{cap}_{\Omega, k}\left(A_{1}\right)=0=\operatorname{cap}_{\Omega^{\mathrm{c}}, k}\left(A_{2}\right)$. Hence the corollary follows from Theorem 1.1.

A similar conclusion can be drawn from Theorem 1.6.1 in [7] if $D(h)$ is a core for $D(k)$. (See also [7], Corollary 4.6.4.)

\section{Examples}

We present several examples of degenerate elliptic operators.

EXAMPLE 3.1. Let $X=\mathbf{R}, \Omega=\langle 0, \infty\rangle, D(h)=W^{1,2}(\Omega) \oplus L_{2}\left(\Omega^{\mathrm{c}}\right)$ and $h(\varphi)=$ $\int_{\Omega}\left|\varphi^{\prime}\right|^{2}$, where $\varphi^{\prime}$ is the distributional derivative. Then $\Omega$ is topologically regular, $|\partial \Omega|=0$ and $h$ is a regular local Dirichlet form. Moreover, $\operatorname{cap}_{\Omega^{\mathrm{c}}}(\partial \Omega)=\operatorname{cap}_{\langle-\infty, 0\rangle}(\{0\})=0$, so $L_{2}(\Omega)$ is $S$-invariant. But $\operatorname{cap}(\partial \Omega)=\operatorname{cap}_{\Omega}(\partial \Omega)=\operatorname{cap}_{\langle 0, \infty\rangle}(\{0\}) \neq 0$. 
EXAMPLE 3.2. Let $X=\mathbf{R}, D(h)=W^{1,2}(\mathbf{R})$ and $h(\varphi)=\int_{\mathbf{R}}\left|\varphi^{\prime}\right|^{2}$. The corresponding operator is the one-dimensional Laplacian. If $\Omega=\mathbf{R} \backslash\{0\}$ then $|\partial \Omega|=0$ and $h$ is a regular local Dirichlet form. Since $\bar{\Omega}^{\mathrm{c}}=\emptyset$ one has cap $\bar{\Omega}^{\mathrm{c}}(\partial \Omega)=0$ and $L_{2}(\Omega)$ is $S$-invariant. The set $\Omega$ is not topologically regular and $\operatorname{cap}(\Omega) \neq 0$. Nevertheless, $H$ is a strongly elliptic operator with constant, and therefore Lipschitz continuous, coefficients. This example shows that a converse of Corollary 2.6 is not valid for degenerate elliptic operators on $\mathbf{R}^{d}$ with coefficients in $W^{1, \infty}\left(\mathbf{R}^{d}\right)$.

The latter remark is of interest since it is proved in [12] that if $h$ is the local regular Dirichlet form obtained as the closure of the form

$$
\varphi \mapsto \sum \int c_{i j} \partial_{i} \varphi \partial_{j} \varphi\left(\varphi \in W^{1,2}\left(\mathbf{R}^{d}\right)\right)
$$

with $c_{i j} \in W^{1, \infty}\left(\mathbf{R}^{d}\right), c_{i j}=c_{j i}$ and $\left(c_{i j}(x)\right) \geq 0$ for all $x \in \mathbf{R}^{d}$, and if $\Omega$ is a Lipschitz domain in $\mathbf{R}^{d}$ such that $L_{2}(\Omega)$ is $S$-invariant, then $\operatorname{cap}(\partial \Omega)=0$. It is unclear whether the Lipschitz property of $\Omega$ could be replaced by the assumption that $\Omega$ is regular in topology.

EXAMPLE 3.3. Let $X=\mathbf{R}, V=\langle-\infty,-1\rangle \cup\langle 0,1\rangle, D(h)=W^{1,2}(V) \oplus L_{2}\left(V^{\mathfrak{c}}\right)$ and $h(\varphi)=\int_{V}\left|\varphi^{\prime}\right|^{2}$. Then $h$ is a local regular Dirichlet form. Choose $\Omega=\langle-1,1\rangle$. Then $\Omega$ is topologically regular. Moreover, $\operatorname{cap}_{\Omega}(\{-1\})=0$ and $\operatorname{cap}_{\bar{\Omega}} \mathrm{c}(\{1\})=0$. Hence $L_{2}(\Omega)$ is $S$-invariant. But $\operatorname{cap}_{\Omega}(\partial \Omega)=\operatorname{cap}_{\Omega}(\{1\}) \neq 0$ and $\operatorname{cap}_{\bar{\Omega}^{c}}(\partial \Omega)=\operatorname{cap}_{\bar{\Omega}^{c}}(\{-1\}) \neq 0$.

EXAMPLE 3.4. Let $X=\mathbf{R}^{2}, \Omega=\langle 0, \infty\rangle \times \mathbf{R}$ the right half-plane and $V=(\langle 0, \infty\rangle \times$ $\langle 0, \infty\rangle) \cup(\langle-\infty, 0\rangle \times\langle-\infty, 0\rangle)$ the union of the first and third quadrants. Let $D(h)=$ $W^{1,2}(V) \oplus L_{2}\left(V^{\mathrm{c}}\right)$ and $h(\varphi)=\int_{V}|\nabla \varphi|^{2}$. Then $h$ is a local regular Dirichlet form. It is easy to verify that there exists an open set $U$ such that the requirements in Condition II of Theorem 2.4 are valid for all $x \in \partial \Omega \backslash\{(0,0)\}$. But if $l$ is the form associated to the Laplacian on $\mathbf{R}^{2}$ then the capacity of the set $\{(0,0)\}$ with respect to the form $l$ is zero. Hence $\operatorname{cap}(\{(0,0)\})=0$ where the capacity is with respect to the form $h$. Therefore the requirements in Condition II of Theorem 2.4 are also valid for $(0,0)$ by adjusting the open set $U$ around $(0,0)$. So $L_{2}(\Omega)$ is $S$-invariant. Alternatively, Condition II of Theorem 1.1 is satisfied with $A_{1}=\{0\} \times\langle-\infty, 0]$ and $A_{2}=\{0\} \times[0, \infty\rangle$.

The next example is a multi-dimensional elliptic operator.

EXAMPLE 3.5. Let $X=\mathbf{R}^{d}$ and $h$ the relaxation, or viscosity closure, of the elliptic form

$$
\varphi \mapsto \sum_{i, j=1}^{d} \int_{\mathbf{R}^{d}} c_{i j}\left(\partial_{i} \varphi\right)\left(\partial_{j} \varphi\right)\left(\varphi \in C_{c}^{\infty}\left(\mathbf{R}^{d}\right)\right),
$$

where the coefficients $c_{i j}=c_{j i} \in L_{\infty}\left(\mathbf{R}^{d}\right)$ are real and the matrix $C=\left(c_{i j}\right)$ is positivedefinite almost everywhere in $\mathbf{R}^{d}$. (The relaxation of a quadratic form is described in [4] 
(see page 28) and the viscosity closure is defined in [6], Section 2.) Then $h$ is again a local regular Dirichlet form (see [5], Theorem 1.1). Assume that $c_{1 i}=0$ for all $i \in\{2, \ldots, d\}$. Moreover, assume that there exists a positive function $\tilde{c} \in C_{b}(\mathbf{R})$ such that $c_{11}(x) \leq \tilde{c}\left(x_{1}\right)$ for almost all $x=\left(x_{1}, \ldots, x_{n}\right) \in \mathbf{R}^{d}$. Let $k$ be the closure of the form $\psi \mapsto \int_{\mathbf{R}} \tilde{c}\left|\psi^{\prime}\right|^{2}$, with $\psi \in C_{c}^{\infty}\left(\mathbf{R}^{d}\right)$. Then $k$ is a local regular Dirichlet form on $L_{2}(\mathbf{R})$. Assume that $\tilde{c}$ is such that $\operatorname{cap}_{\langle 0, \infty\rangle, k}(\{0\})=0$. If $\psi \in D(k)$ and $\tau \in C_{c}^{\infty}\left(\mathbf{R}^{d-1}\right)$ then $\psi \otimes \tau \in D(h)$ and one has an estimate

$$
\|\psi \otimes \tau\|_{D(h)} \leq M\|\varphi\|_{D(k)}\|\tau\|_{W^{1,2}\left(\mathbf{R}^{d-1}\right)}
$$

where $M=1+\max _{i, j}\left\|c_{i j}\right\|_{\infty}$. Therefore setting $\Omega=\langle 0, \infty\rangle \times \mathbf{R}^{d-1}$ one deduces that $\operatorname{cap}_{\Omega}(\partial \Omega)=\operatorname{cap}_{\Omega}\left(\{0\} \times \mathbf{R}^{d-1}\right)=0$. Hence one can choose $A_{1}=\partial \Omega$ in Condition II of Theorem 1.1 and $L_{2}(\Omega)$ is invariant under the semigroup corresponding to $h$.

One can construct more complicated examples by combination with the idea underlying Example 3.4.

Assume that there exist positive functions $\tilde{c}_{1}, \tilde{c}_{2} \in C_{b}(\mathbf{R})$ such that

$$
c_{11}(x) \leq \begin{cases}\tilde{c}_{1}\left(x_{1}\right) & \text { if } x_{2}>0 \\ \tilde{c}_{2}\left(x_{1}\right) & \text { if } x_{2}<0\end{cases}
$$

for almost all $x \in \mathbf{R}^{d}$. Let $k_{1}$ and $k_{2}$ denote the Dirichlet forms on $L_{2}(\mathbf{R})$ with coefficients $\tilde{c}_{1}$ and $\tilde{c}_{2}$, respectively. Suppose $\operatorname{cap}_{\langle 0, \infty\rangle, k_{1}}(\{0\})=0$ and $\operatorname{cap}_{\langle-\infty, 0\rangle, k_{2}}(\{0\})=0$. Then one may choose $A_{1}=\{0\} \times[0, \infty\rangle \times \mathbf{R}^{d-2}$ and $A_{2}=\{0\} \times\langle-\infty, 0] \times \mathbf{R}^{d-2}$ in Condition II of Theorem 1.1, and $L_{2}(\Omega)$ is again invariant under the semigroup corresponding to $h$.

Finally we note that these examples can all be extended by application of the domination principle given by Corollary 2.11 .

The regularity of the form $k$ in Corollary 2.11 is essential as the next example shows.

EXAMPLE 3.6. Let $X=\langle 0,1\rangle \cup\langle 2,3\rangle, D(h)=W_{0}^{1,2}(X)$ and $h(\varphi)=\int_{X}\left|\varphi^{\prime}\right|^{2}$. Define the form $k$ by

$$
D(k)=\left\{\varphi \in W^{1,2}(X): \varphi(0)=\varphi(3) \text { and } \varphi(1)=\varphi(2)\right\}
$$

and $k(\varphi)=\int_{X}\left|\varphi^{\prime}\right|^{2}$. Then $h \leq k$. Let $S, T$ be the associated semigroups on $L_{2}(X)$. Choose $\Omega=\langle 0,1\rangle$. Then $\Omega$ is $S$-invariant, but not $T$-invariant.

\section{Acknowledgement}

Parts of this work were carried out during a visit of the first named author to the Australian National University and the second named author to the University of Auckland. The authors wish to thank the referee for drawing our attention to Proposition 2.1 and suggesting Example 3.6. They also thank Wolfgang Arendt for several useful comments. 


\title{
REFERENCES
}

[1] ARENDT, W. AND WARMA, M., The Laplacian with Robin boundary conditions on arbitrary domains, Potential Anal. 19 (2003), 341-363.

[2] - Dirichlet and Neumann boundary conditions: What is in between?, J. Evol. Equ. 3 (2003), 119-135.

[3] BouleaU, N. AND HirSCH, F., Dirichlet forms and analysis on Wiener space, vol. 14 of de Gruyter Studies in Mathematics. Walter de Gruyter \& Co., Berlin, 1991.

[4] Dal Maso, G., An introduction to $\Gamma$-convergence, vol. 8 of Progress in Nonlinear Differential Equations and their Applications. Birkhäuser Boston Inc., Boston, MA, 1993.

[5] Elst, A. F. M. Ter, Robinson, D. W., Sikora, A. And Zhu, Y., Dirichlet forms and degenerate elliptic operators, In KoElink, E., NeERVEn, J. VAn, PAGTER, B. DE AND SwEERS, G., eds., Partial Differential Equations and Functional Analysis, vol. 168 of Operator Theory: Advances and Applications. Birkhäuser, 2006, 73-95. Philippe Clement Festschrift.

[6] - Second-order operators with degenerate coefficients, Proc. London Math. Soc. 95 (2007), $299-328$.

[7] Fukushima, M., Oshima, Y. AND TAKEDA, M., Dirichlet forms and symmetric Markov processes, vol. 19 of de Gruyter Studies in Mathematics. Walter de Gruyter \& Co., Berlin, 1994.

[8] HeINONEN, J., Lectures on analysis and metric spaces. Universitext. Springer, New York, 2001.

[9] NAGEL, R., ed., One-parameter semigroups of positive operators, Lecture Notes in Mathematics 1184, Berlin etc., 1986. Springer-Verlag.

[10] OuHabAZ, E.-M., Analysis of heat equations on domains, vol. 31 of London Mathematical Society Monographs Series. Princeton University Press, Princeton, NJ, 2005.

[11] REED, M. AND Simon, B., Methods of modern mathematical physics IV. Analysis of operators. Academic Press, New York etc., 1978.

[12] Robinson, D. W. And SIKora, A., Degenerate elliptic operators: capacity, flux and separation, J. Ramanujan Math. Soc. 22 (2007), 385-408.

[13] Schmuland, B., On the local property for positivity preserving coercive forms. In MA, Z. M. AND RÖCKNER, M., eds., Dirichlet forms and stochastic processes. Walter de Gruyter \& Co., Berlin, 1995 , 345-354. Papers from the International Conference held in Beijing, October 25-31, 1993, and the School on Dirichlet Forms, held in Beijing, October 18-24, 1993.

\author{
A. F. M. ter Elst \\ Department of Mathematics \\ University of Auckland \\ Private bag 92019 \\ Auckland \\ New Zealand \\ Derek W. Robinson \\ Centre for Mathematics and its Applications \\ Mathematical Sciences Institute \\ Australian National University \\ Canberra, ACT 0200 \\ Australia
}

